\title{
BMJ Open Quality of life of elderly patients with solid tumours undergoing adjuvant cancer therapy: a systematic review
}

\author{
Karis Kin-Fong Cheng, ${ }^{1}$ Ethel Yee-Ting Lim, ${ }^{1}$ Ravindran Kanesvaran ${ }^{2}$
}

To cite: Cheng KK-F, Lim EYT, Kanesvaran R. Quality of life of elderly patients with solid tumours undergoing adjuvant cancer therapy: a systematic review. BMJ Open 2018;8:e018101. doi:10.1136/ bmjopen-2017-018101

- Prepublication history and additional material for this paper are available online. To view these files, please visit the journal online (http://dx.doi. org/10.1136/bmjopen-2017018101).

Received 7 June 2017 Revised 31 0ctober 2017 Accepted 9 November 2017

Check for updates

${ }^{1}$ Alice Lee Centre for Nursing Studies, Yong Loo Lin School of Medicine, National University of Singapore, National University Health System, Singapore,

Singapore

${ }^{2}$ National Cancer Centre

Singapore, Singapore, Singapore

Correspondence to

Professor Karis Kin-Fong Cheng; nurckfk@nus.edu.sg

\begin{abstract}
Objectives The measurement of quality of life (QoL) in elderly cancer population is increasingly being recognised as an important element of clinical decision-making and the evaluation of treatment outcome. This systematic review aimed to summarise the evidence of QoL during and after adjuvant therapy in elderly patients with cancer. Methods A systematic search was conducted of studies published in CINAHL plus, CENTRAL, PubMed, PsycINFO and Web of Science from the inception of these databases to December 2016. Eligible studies included RCTs and non-RCTs in which QoL was measured in elderly patients (aged 65 years or above) with stage I-III solid tumours who were undergoing adjuvant chemotherapy and/ or radiotherapy. Because of the heterogeneity and the insufficient data among the included studies, the results were synthesised narratively.

Results We included 4 RCTs and 14 non-RCTs on 1785 participants. In all four RCTs, the risk of bias was low or unclear for most items but high for detection. Of the 14 non-RCTs, 5 studies were judged to have a low or moderate risk of bias for all domains, and the other 9 studies had a serious risk of bias in at least one domain. The bias was observed mainly in the confounding and in the selection of participants for the study. For most elderly patients with breast cancer, the non-significant negative change in the QoL was transient. A significant increase in the QoL during the course of temozolomide in elderly patients with glioblastoma but a decreasing trend in QoL after radiotherapy was shown. This review also shows a uniform trend of stable or improved QoL during adjuvant therapy and at follow-up evaluations across the studies with prostate, colon or cervical cancer population. Conclusions This review suggests that adjuvant chemotherapy and radiotherapy may not have detrimental effects on QoL in most elderly patients with solid tumours.
\end{abstract}

\section{INTRODUCTION}

In many countries, the incidence of cancer among older people is increasing. This increase can be attributed to the remarkable growth of the elderly demographic and the common pathophysiology of cancer and ageing. ${ }^{12}$ As a result, the demands for and the importance of broadening clinical trials to include older adults, incorporating geriatric-specific end points ${ }^{3}$ and integrating

\section{Strengths and limitations of this study}

- A systematic search of the published literature in major databases from their inception to December 2016 was conducted.

- The risk of bias and the methodological aspects of quality of life reporting in the included studies were assessed.

- The search of grey literature, unpublished studies, ongoing clinical trials and theses and dissertations were not conducted.

- The studies included in this review are mainly nonrandomised controlled trials.

- The meta-analysis was not conducted to pool the data and the Grading of Recommendations Assessment, Develooment and Evaluation (GRADE) approach was not used to assess the quality of evidence of the included studies.

geriatric assessment to address the needs of individuals are also increasing. ${ }^{4}$ Although quality of life (QoL) is not formally a part of the geriatric assessment, the measurement of QoL in the elderly cancer population is increasingly being recognised as an important patient-reported outcome to complement the clinician's evaluation of disease progression and the determination of the clinical benefit and the burden of cancer treatment, along with toxicity, survival and mortality rates. QoL is also considered a useful outcome measure to enhance patient-clinician communication and patient compliance in elderly patients with breast cancer during cancer treatment. ${ }^{5}$ In a short literature review, Wedding et al reported that elderly patients with cancer tend to perceive their QoL as more important than gains in survival when compared with younger patients. ${ }^{6}$ Nevertheless, our understanding of the effect of cancer treatment on the QoL of elderly patients remains very limited. Clinically, the decisions regarding cancer therapy and the clinical management of elderly patients with cancer may be complicated by their vulnerability to chemotoxicity and the pathological changes of ageing 
together with different considerations of the treatment benefit and harm margins, functional decline, tolerability and QoL issues. A univariate analysis by Extermann et al revealed an association of the QoL effect with dose modification of chemotherapy in older patients. ${ }^{7}$ The literature states that elderly patients with cancer are less likely than their younger counterparts to be treated with a full course of adjuvant chemotherapy and radiotherapy. ${ }^{8}$ Consideration should be given to approaches that can prolong life expectancy, but not at the expense of QoL and physical and psychological functioning. For cancers with an extremely poor prognosis, such as glioblastoma, the extension of survival is less clinically meaningful if the patient has a decline in QoL. ${ }^{9}$ Researchers have also suggested that QoL be used as the main end point to support clinical decision-making if different cancer treatments are equally effective in terms of survival. ${ }^{10}$ To the best of our knowledge, a systematic review of the effects of adjuvant therapy on the QoL of elderly patients with cancer has not yet been published. Therefore, we undertook a systematic review of the literature to summarise the evidence of global or overall QoL and other domains pertaining to QoL during and after adjuvant therapy in elderly patients with stage I-III solid tumours. We considered the following question: 'Does the global or overall QoL during and after adjuvant chemotherapy and/or radiotherapy decline, maintain or improve from baseline in elderly patients with solid tumours in randomised controlled trials (RCTs) or non-RCTs?' In this review, QoL refers to the health-related QoL of elderly patients, considering the corresponding global, physical, cognitive, psychological and social domains as affected by the adjuvant therapy.

\section{METHODS}

The methodology of this systematic review included a prespecified literature search strategy, inclusion and exclusion criteria, process for selecting studies, assessment of methodological quality of studies and data synthesis. The review protocol was not registered in an international registery. The conduct and reporting of this systematic review were in accordance with the planned review methods except for the addition of assessment of risk of bias (RoB) of the included studies using the Cochrane Risk of Bias tool for RCTs and Risk of Bias tool in Non-Randomised Studies of Interventions (ROBINS-I) for non-RCTs.

\section{Literature search strategy}

A systematic electronic search of peer-reviewed English-language articles published in CINAHL plus (1937-2016), CENTRAL (1993-2016), PubMed (19962016), PsycINFO (1967-2016) and Web of Science (19002016) from the inception of these databases to December 2016 was conducted. The date last searched was in March 2017. Searches were limited to human studies published in English. A pilot search on CINAHL was performed to identify the relevant keywords contained in the title, abstract and subject descriptors. Three broad categories of concepts were searched: 'elderly', 'cancer' and 'quality of life'. The search terms included (older* OR elder* OR geriatric OR gerontology* OR senior OR aged) AND (oncology OR cancer* OR neoplasm*) AND (quality of life OR QOL). The full electronic search strategy is presented in Appendix A. The reference lists of the included articles were also examined to identify additional eligible articles.

\section{Study selection}

\section{Inclusion criteria}

We included RCTs and non-RCTs in which QoL was measured in elderly patients (aged 65 years or above) with stage I-III solid tumours who were undergoing adjuvant chemotherapy and/or radiotherapy. Non-RCTs include quantitative studies such as observational, before-andafter and longitudinal studies, in which the allocation of intervention (analogy of treatment) occurs during the course of the usual treatment decisions. ${ }^{11}{ }^{12}$ We required that the baseline and at least one global or overall $\mathrm{QoL}$ data element during and/or after adjuvant chemotherapy and/or radiotherapy be collected and reported in the studies so as to allow an in-context comparison of before and after adjuvant therapy. Studies that covered heterogeneous age groups were included if a subgroup analysis was performed and reported for those aged 65 years or above.

\section{Exclusion criteria}

Studies were excluded if they involved patients with haematological malignancies, distant metastatic cancer or recurrent cancer without a separate analysis and report of solid tumours or non-metastatic/regional metastatic cancer. We also excluded case reports, qualitative studies, literature reviews, studies that evaluated surgical or procedure-related treatment and presented in abstract form.

\section{Process for selecting studies}

We screened articles obtained from keyword searching for duplicates electronically with End-Note and then manually. After duplicate removal, we assessed the remaining articles for eligibility based on titles and abstracts. We included studies in full-text screening if they were RCTs or non-RCTs, included elderly patients with stage I-III solid tumours who were undergoing adjuvant chemotherapy and/or radiotherapy, and reported QoL. We retrieved full-text articles if we considered the studies relevant and if there was insufficient information to determine eligibility. We then examined each full-text article against the inclusion and exclusion criteria of the review.

\section{Data extraction}

We extracted data related to publication information, sample characteristics, type of cancer, type of adjuvant chemotherapy and/or radiotherapy, supportive care, QoL measurements and results, dropouts and authors' conclusions. Functional status and comorbidities at baseline and 
therapy-related adverse effects (where reported) were also extracted because of concern that they might co-vary or confound with those of adjuvant therapy to alter the change of QoL.

\section{Assessment of methodological quality of studies on QoL}

The methodological quality of the included studies on QoL was assessed using a checklist of predefined criteria for studies on QoL. ${ }^{11} 12$ The checklist was originally developed to assess the internal and external validity of prognostic studies ${ }^{13}$ and was modified to assess the methodological aspects of QoL reporting in later studies. ${ }^{11} 12$ The checklist covers the following 14 items: sampling (two items), selection of QoL measurement (one item), data collection process (two items), response rate (two items), group comparison (one item), clarity of reporting (five items) and determination of prognostic factors (one item), all of which are important in QoL studies. For each item, a score of 1 or 0 was given; 1 was assigned to an item meeting the methodological criteria, while 0 was assigned if an item neither met the criteria nor described the related parameter sufficiently. The possible score ranged from 0 to 14 , with scores of 10 or above, 7 to 9 and 6 or less indicating high, moderate and low quality, respectively. ${ }^{11}$

\section{Assessment of risk of bias}

The RoB of the included studies was evaluated using the Cochrane RoB tool and ROBINS-I for RCTs and non-RCTs, respectively. ${ }^{14}{ }^{15}$ Both tools are domain-based evaluations of $\mathrm{RoB}$ with respect to the internal validity of studies. The Cochrane RoB tool covers the domains of selection, performance, detection, attrition and reporting bias, and other sources of bias. A judgement of 'yes' indicates a low RoB; 'no', a high RoB and 'unclear', either an unclear or unknown RoB. ${ }^{15}$ The ROBINS-I tool covers seven domains: bias due to confounding; bias in selection of participants into the study; bias in classification of interventions; bias due to deviations from intended interventions; bias due to missing data; bias in measurement of outcomes and bias in selection of the reported results. The RoB judgements within each domain are categorised as 'low risk' if the study is comparable to a well-performed RCT, 'moderate risk' if the study is sound but cannot be considered comparable to a well-performed RCT, 'serious risk' if the study has some considerable problems, 'critical risk' if the study is too problematic and 'no information'. The judgements within each domain contribute to the overall RoB. ${ }^{14}$

In this review, two reviewers (LEYT and TDRL) independently performed the literature search, eligibility assessments and study selection. The data extraction, methodological quality assessment and the RoB evaluation were conducted by CKKF and LEYT. Discrepancies and disagreements were discussed and resolved by consensus.

\section{Data synthesis}

Because of the variations in study design, cancer populations and QoL scales and the insufficient data among the included studies, a meta-analysis was deemed impossible, and the results were synthesised narratively taking into account of the RoB of individual studies. In addition, we report a change in QoL scores from baseline to the middle of and to the completion of adjuvant therapy, and to the post-treatment follow-up period of individual studies where data were available. We defined ' 0 ' as no change, ' $\uparrow$ ' denotes better QoL than baseline and ' $\downarrow$ ' represents worse QoL than baseline. The effect size (ES) was also calculated for individual studies for which sufficient information was available: 0.2 to $<0.5$ was considered small, 0.5 to $<0.8$ moderate and $\geq 0.8$ large.

\section{RESULTS}

\section{Search results}

The initial search identified 56935 articles, of which 440 were considered potentially relevant after checking for duplicates and title and abstract screening. After full-text assessment of the 440 articles, 18 met the eligibility criteria for inclusion in the review and analysis (figure 1). ${ }^{16-33} \mathrm{In}$ most cases, the articles were excluded mainly because of the lack of QoL assessment during adjuvant therapy, a separate report of participants aged 65 years or above and/or a separate report of the QoL of participants who were undergoing adjuvant therapy or suffering from non-metastatic cancer.

\section{Description of studies}

Eleven studies were published between 2000 and 2009, and seven between 2010 and 2015. With respect to the country of origin, 10 were from Europe, 4 from the USA, 2 from South Korea and 1 from Canada; the other was a multicountry study. As for the study design, 13 studies were non-RCTs (before-and-after or longitudinal studies) that assessed the QoL of patients who were undergoing adjuvant chemotherapy, ${ }^{17} 19202224303133$ radiotherapy $^{16} 32$ or concomitant chemotherapy and radiotherapy. ${ }^{26-28}$ Four were RCTs ${ }^{18} 22^{25}$; two of these compared the effects of different chemotherapy regimens on QoL, one study compared the effects of chemotherapy and hormonal therapy against those of hormonal therapy alone on QoL and the other compared the effects of radiotherapy and supportive care with those of supportive care alone on QoL. One was a validation study that involved a QoL evaluation of participants who were undergoing radiotherapy with or without hormonal therapy ${ }^{29}$ (table 1 ).

The sample size of participants aged 65 years or older was reported by 17 of the 18 studies $^{16-3133}$; Caffo et al did not separately report the number of participants aged 65 years and older. ${ }^{32}$ The sample sizes ranged from 11 to 368 per study. ${ }^{16-31}{ }^{33}$ In all, these 17 studies included 1785 participants; 764 participants from RCTs and 1021 participants from non-RCTs. ${ }^{16-31} 33$ Of these 1785 participants, 1633 completed the baseline QoL questionnaire; 671 participants from RCTs and 962 participants from non-RCTs. Furthermore, the baseline completion rates ranged from $64.7 \%$ to $100 \%$. Where reported, the age 
56,935 records identified through database searching

CINAHL Plus: 4609

Web of Science: 12,252

CENTRAL: 7831

PsycINFO: 9917

PubMed: 22,326
1 additional record identified through other source

\section{3,421 of records after duplicates removed}

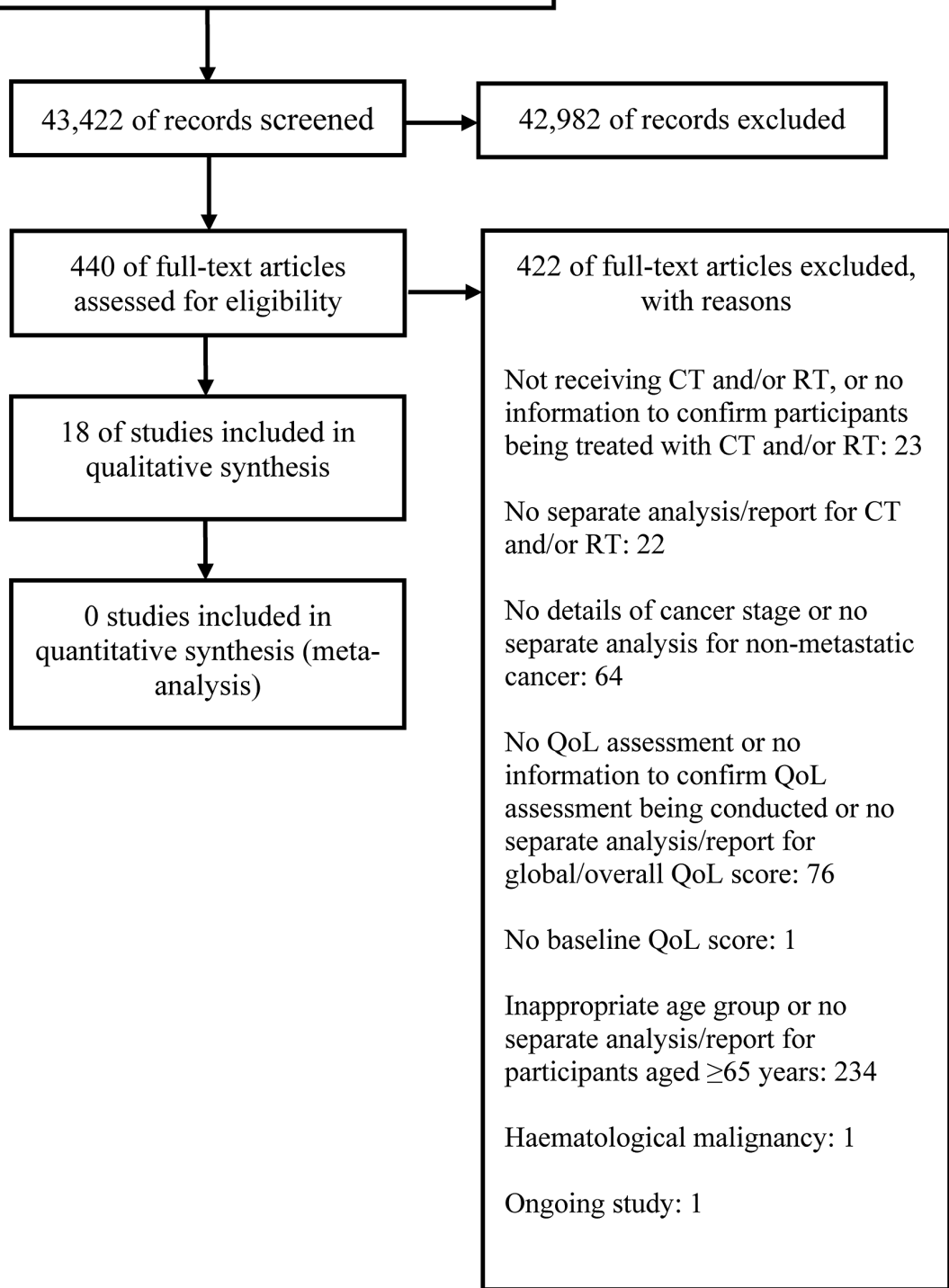

Figure 1 Study flow diagram. CT, chemotherapy; QoL, quality of life; RT, radiotherapy.

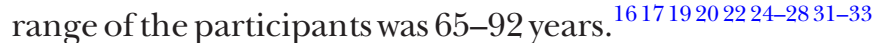
Eleven studies included participants aged 80 years and

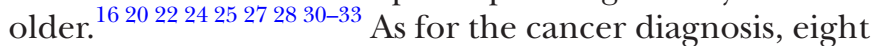
studies included participants with breast cancer, ${ }^{16-23}$ four studies focused on glioblastoma participants ${ }^{24-27}$ and two studies considered participants with colon cancer. ${ }^{30}{ }^{31} \mathrm{We}$ included one study each on mixed, ${ }^{28}$ prostate, ${ }^{29}$ cervical $^{32}$ and lung cancer ${ }^{33}$ participants.
The most frequently used QoL instrument was the European Organization for Research and Treatment of Cancer general questionnaire (EORTC QLQ-C30; 13 studies). ${ }^{16} 1721-3133$ Nine studies also used a disease-specific QoL instrument along with the EORTC QLQ-C30 for breast, ${ }^{161721-23}$ brain $^{242527}$ and lung ${ }^{33}$ cancer populations. The follow-up QoL evaluation was conducted at various intervals during adjuvant therapy and the post-treatment 


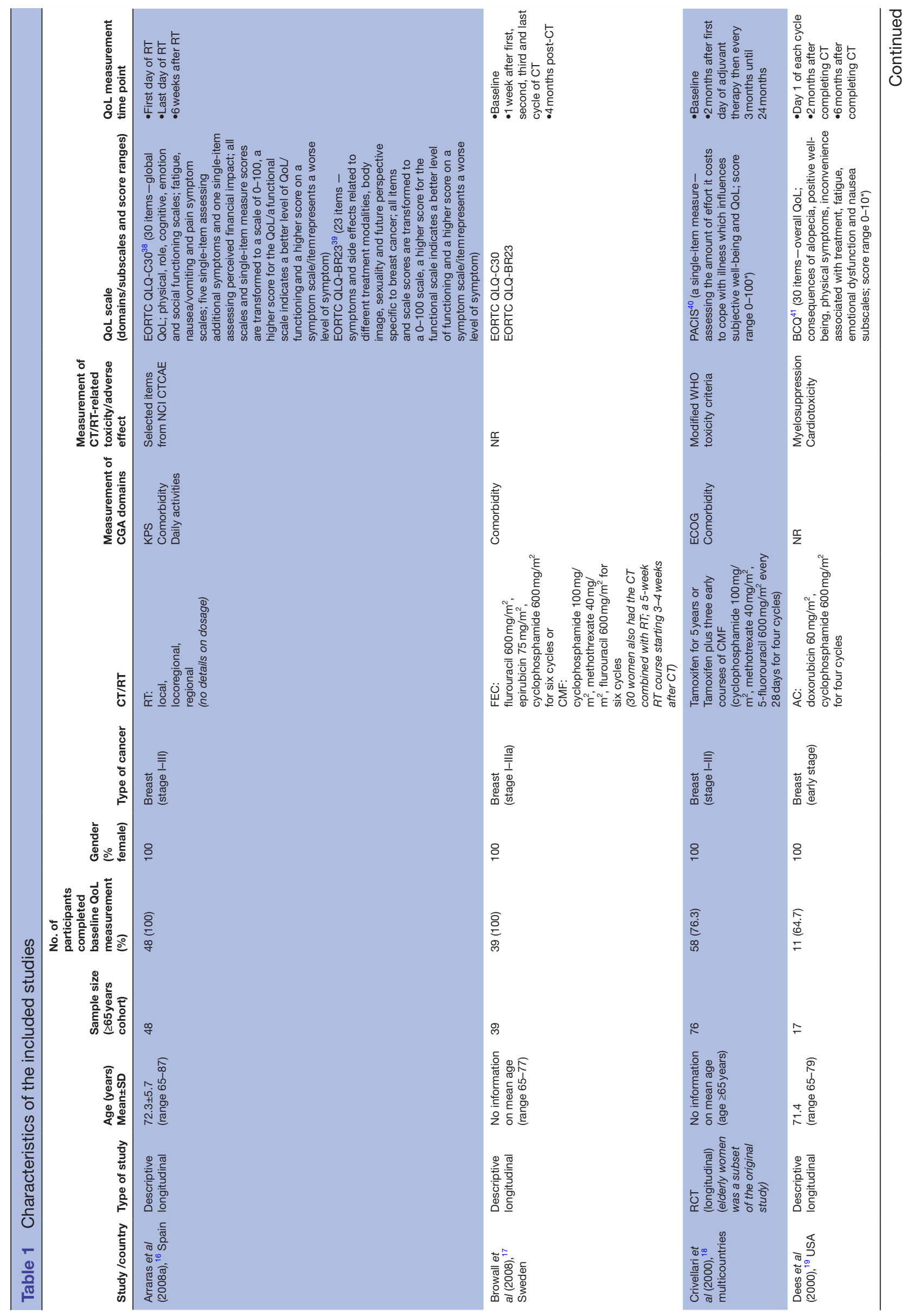




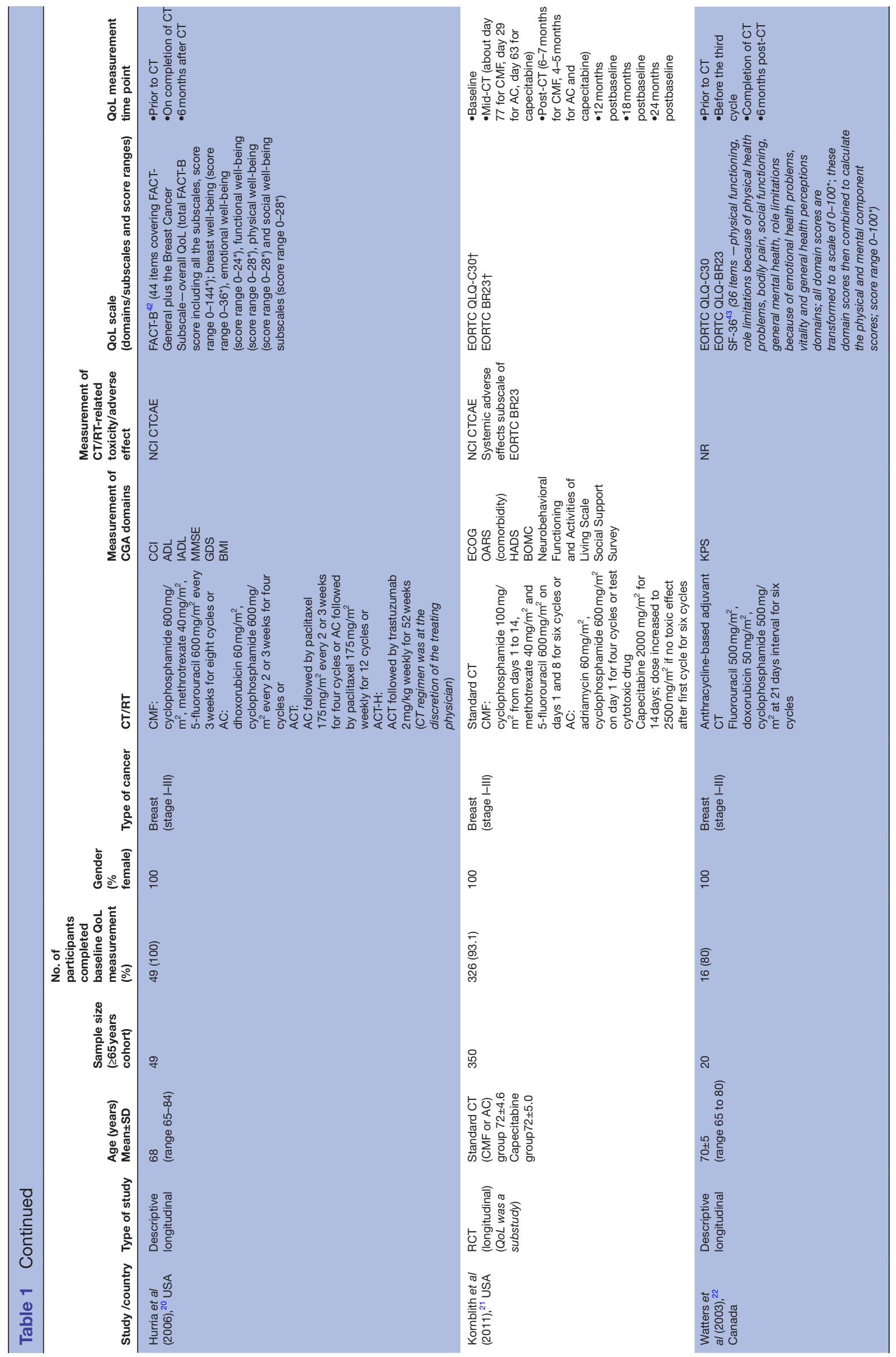




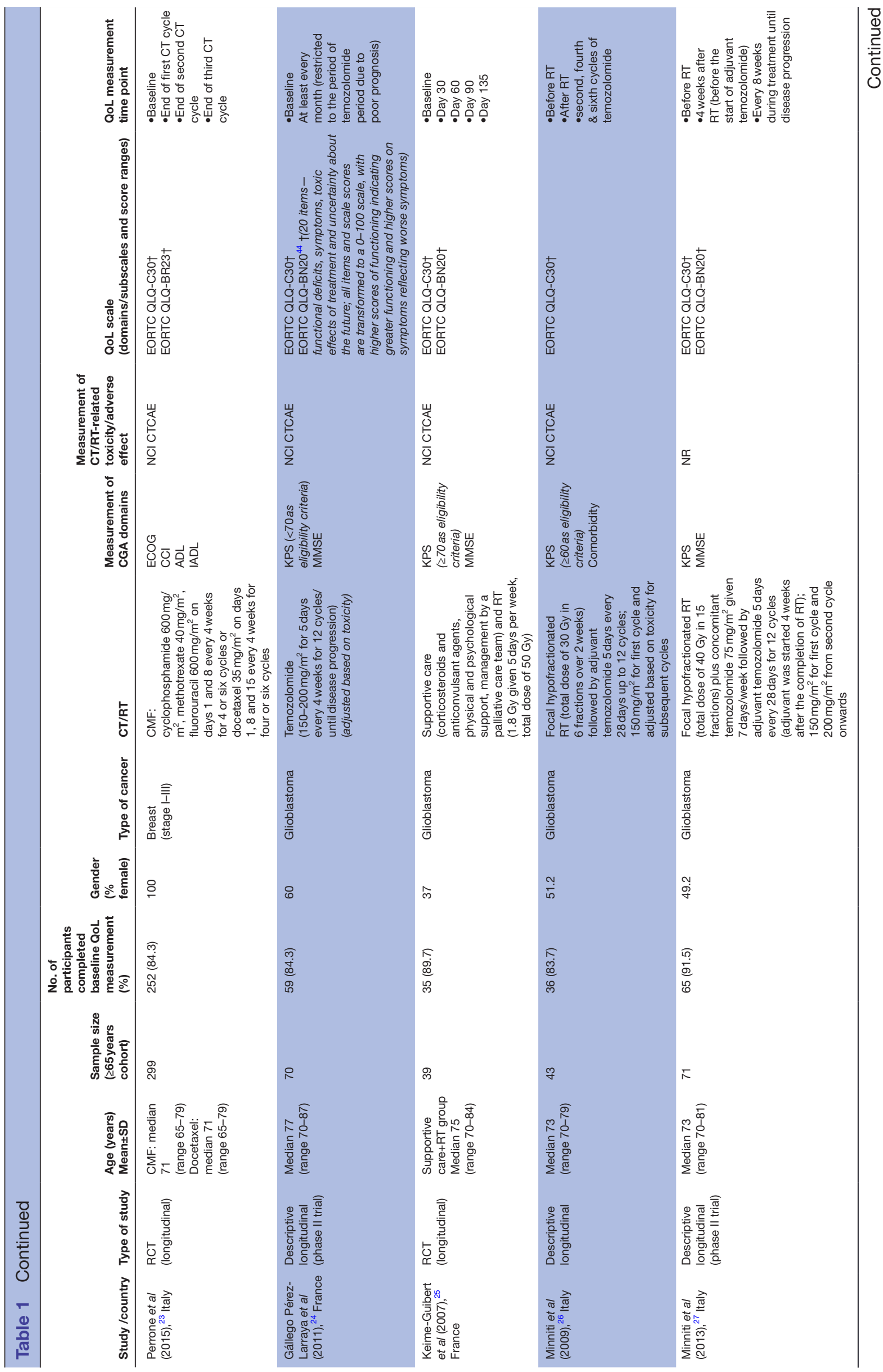




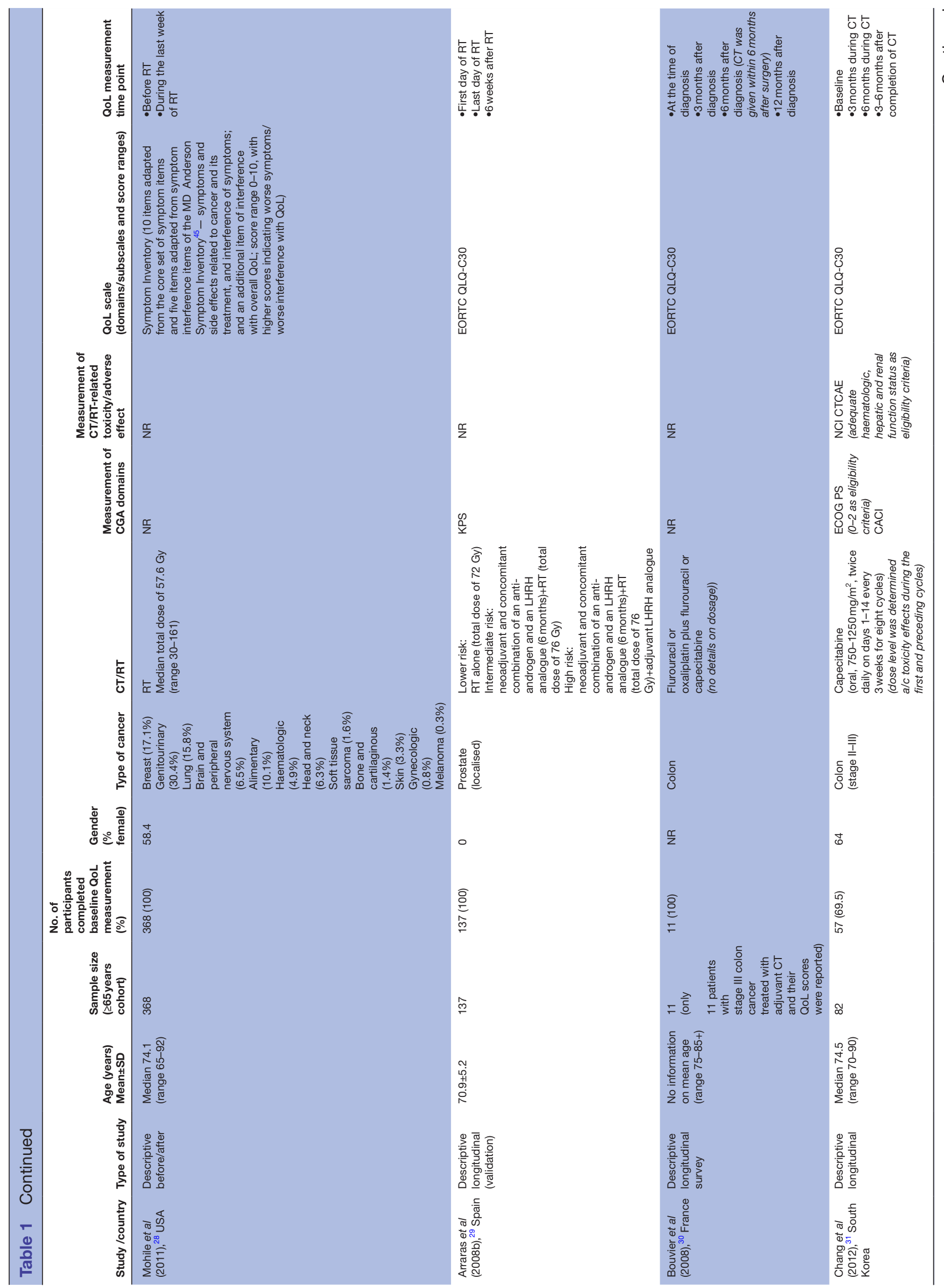




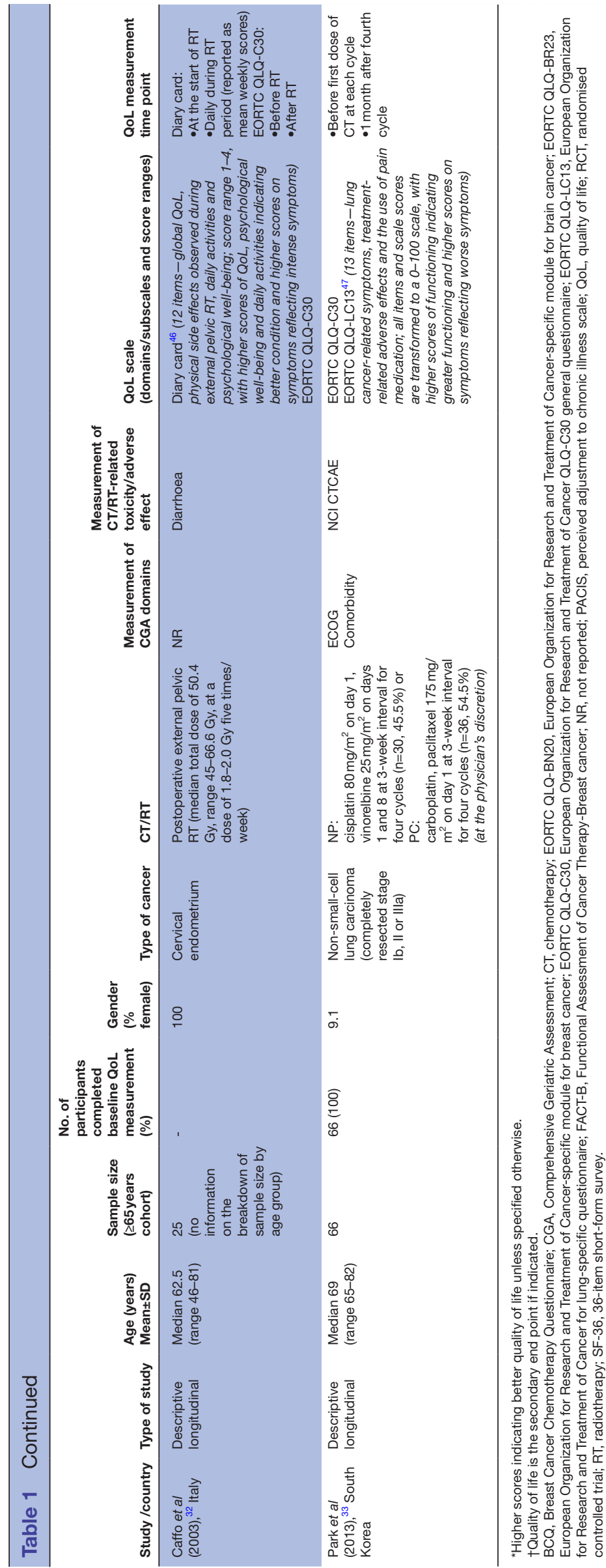


period. Ten studies reported at least one QoL evaluation during adjuvant therapy, ${ }^{17-19}$ 21-25 3132 and five evaluated QoL immediately after the completion of adjuvant therapy. ${ }^{20-22} 2829$ The timing of the QoL evaluation after adjuvant therapy ranged from 1 month after treatment to 24 months after the first day of adjuvant therapy. Ten studies followed participants for 6 months or less after the completion of adjuvant therapy. ${ }^{16} 171920222529-3133$ Two studies included a QoL evaluation of 24 months after the first day of chemotherapy. ${ }^{1821}$

The geriatric domains of functional status and/or comorbidities at baseline were examined and reported in 14 studies. ${ }^{16-18}$ 20-27 293133 As shown in table 2, two studies reported the mean score of the Karnofsky Performance Scale (KPS) as 90 or above, ${ }^{16}{ }^{29}$ whereas three reported the median score of the KPS as 70 or above at baseline. ${ }^{25-27}$ A KPS score of $<70$ was used as a cut-off for the recruitment criterion in one study. ${ }^{24}$ Comorbid conditions were reported in eight studies ${ }^{16} 17202123263133$; six of these involved participants with a limiting comorbidity or with three or more comorbidities. ${ }^{16172123} 3133$ Twelve studies measured cancer therapy-related toxicity during adjuvant therapy, ${ }^{16}$ 18-21 23-26 31-33 and nine of these used National Cancer Institute's Common Terminology Criteria for Adverse Events. ${ }^{16} 202123-263133$ With respect to haematological toxicity, two studies reported grade 3 or 4 toxicity in fewer than $10 \%$ of participants, ${ }^{18} 31$ and five reported such toxicity in $25 \%$ or higher during adjuvant chemotherapy or concomitant radiotherapy and chemotherapy. ${ }^{20} 23242633$ With respect to non-haematological toxicity, a study reported grade 3 or 4 toxicity in fewer than $10 \%$ of participants, ${ }^{18}$ and four reported such toxicity in $25 \%$ or higher during adjuvant chemotherapy or concomitant radiotherapy and chemotherapy ${ }^{2023261}$ (table 2).

\section{Methodological quality}

Thirteen studies attained scores of 10 or higher (high quality), ${ }^{16-2733}$ three scored 7-9 (moderate quality) 283031 and two scored 6 or lower (low quality). ${ }^{29}{ }^{32}$ The main methodological drawbacks of the included studies were the lack of determination of the prognostic factors for QoL (100\%) and the lack of data on the time since diagnosis or treatment $(77.8 \%)$ and the characteristics of non-responders $(77.8 \%)$ (table 3 ).

\section{Risk of bias}

\section{Randomised controlled trials}

In all four RCTs, the RoB was low or unclear for most items but high for detection because of the subjective and self-reporting nature of the QoL assessment. One RCT did not blind the participants and staff and thus was judged to have a high risk of performance bias. ${ }^{18}$ The remaining three RCTs did not report information on the blinding of participants and personnel to allow for a judgement of the performance bias. ${ }^{21} 2325$ We judged three RCTs to have an unclear risk of attrition bias because of the lack of explicit information on patients' lost to follow-up and missing data ${ }^{182125}$ (figure 2).

\section{Non-randomised controlled trials}

Of the 14 non-RCTs, five studies were judged to have a low or moderate RoB for all domains, ${ }_{16} 20263133$ and the other nine studies had a serious RoB in at least one domain. ${ }^{1719222428-3032}$ The bias were observed mainly in the confounding, in the selection of participants for the study and in the measurement of outcomes. Although most of the studies measured some confounding factors (eg, functional performance status or comorbidity) at baseline, no stratification in the study design or adjustment in the data analysis was made to control their effects. ${ }^{16} 172022-2427293133$ Four non-RCTs did not measure functional performance status or comorbidities at baseline. ${ }^{19} 283032$ The bias in the selection of participants was either moderate or serious in all the non-RCTs. ${ }^{16} 171920$ 22-24 27-33 Only fit and functional elderly patients seemed to have been enrolled in these studies, and hence, the study cohorts might not be representative of the real-world population. Like the RCTs, all 14 non-RCTs had a moderate-to-serious RoB in the measurement outcomes because of the subjective and self-reporting nature of the QoL assessment. The bias in the selection of reported results was unclear in all the non-RCTs because of unavailability of study protocols $^{1617192022-2427-33}$ (table 4).

\section{QoL outcomes}

Breast cancer

\section{EORTC-QLQ-C3O}

Three studies reported the global QoL scores at baseline, during chemotherapy, at the time of completion of chemotherapy and 4-12 months after the completion of chemotherapy. ${ }^{172} 22$ The participants in these studies were treated with the standard chemotherapy regimen for breast cancer, including an anthracycline-based, cyclophosphamide/ methotrexate/fluorouracil (CMF) or fluorouracil/epirubicin/cyclophosphamide regimen. In the study by Kornblith $e t a l^{21}$ approximately half of the participants received capecitabine. Browall et al reported statistically significantly lower global QoL scores during (ES, 0.74) and immediately after the completion (ES, 0.71) of chemotherapy than at baseline and a non-significant decline in the global QoL score 4 months after chemotherapy. ${ }^{17}$ Watters et al also revealed a statistically significantly lower global QoL score immediately after the completion of chemotherapy (ES, 0.66) than at baseline and a non-significant decline in the global QoL scores during and 6 months after chemotherapy. ${ }^{22}$ Browall et al and Watters et al also reported the domain scores, wherein statistically significantly lower scores in the role and social functioning domains were found immediately after the completion of chemotherapy than at baseline. No significant reductions in role and social wellbeing were reported during or 4-6 months after the completion of chemotherapy. ${ }^{1722}$ Emotion was the only domain that showed an improvement from baseline to the follow-up evaluations, with a statistically significantly higher score during chemotherapy. The domains of physical and cognitive functioning revealed no statistically significant differences across time. ${ }^{1722}$ In the study by Kornblith $e t$ al, both 


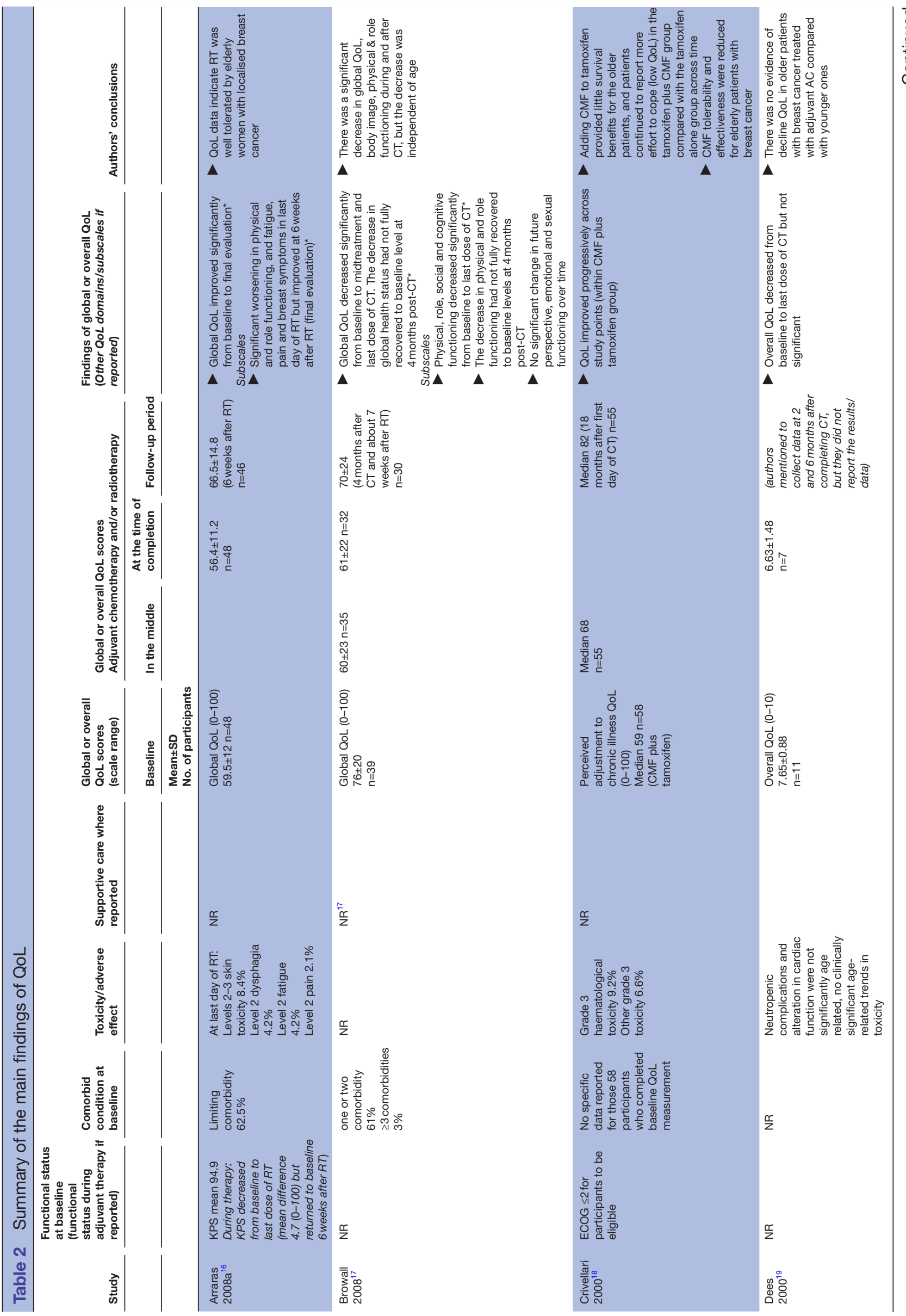

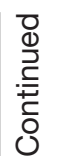




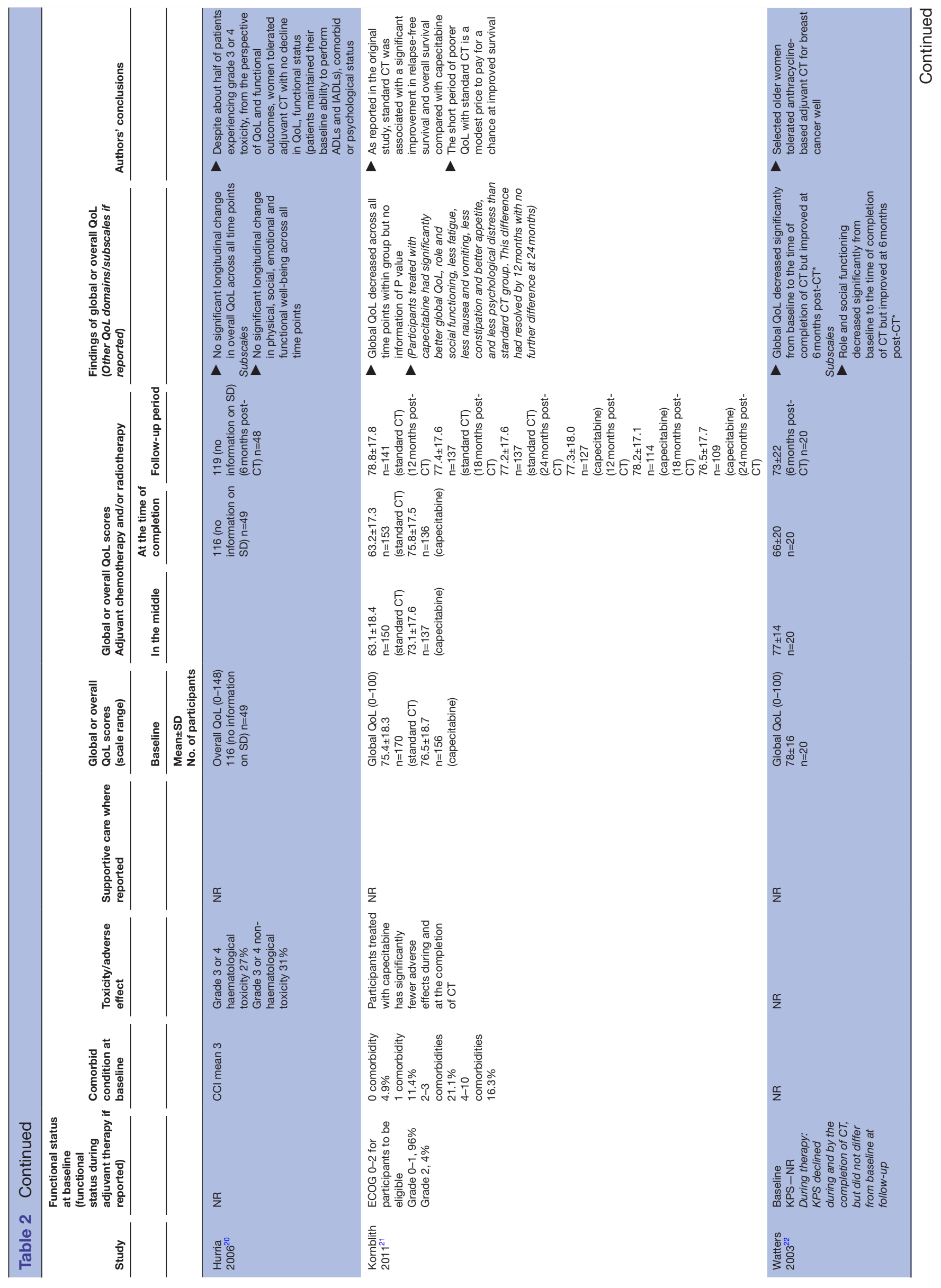




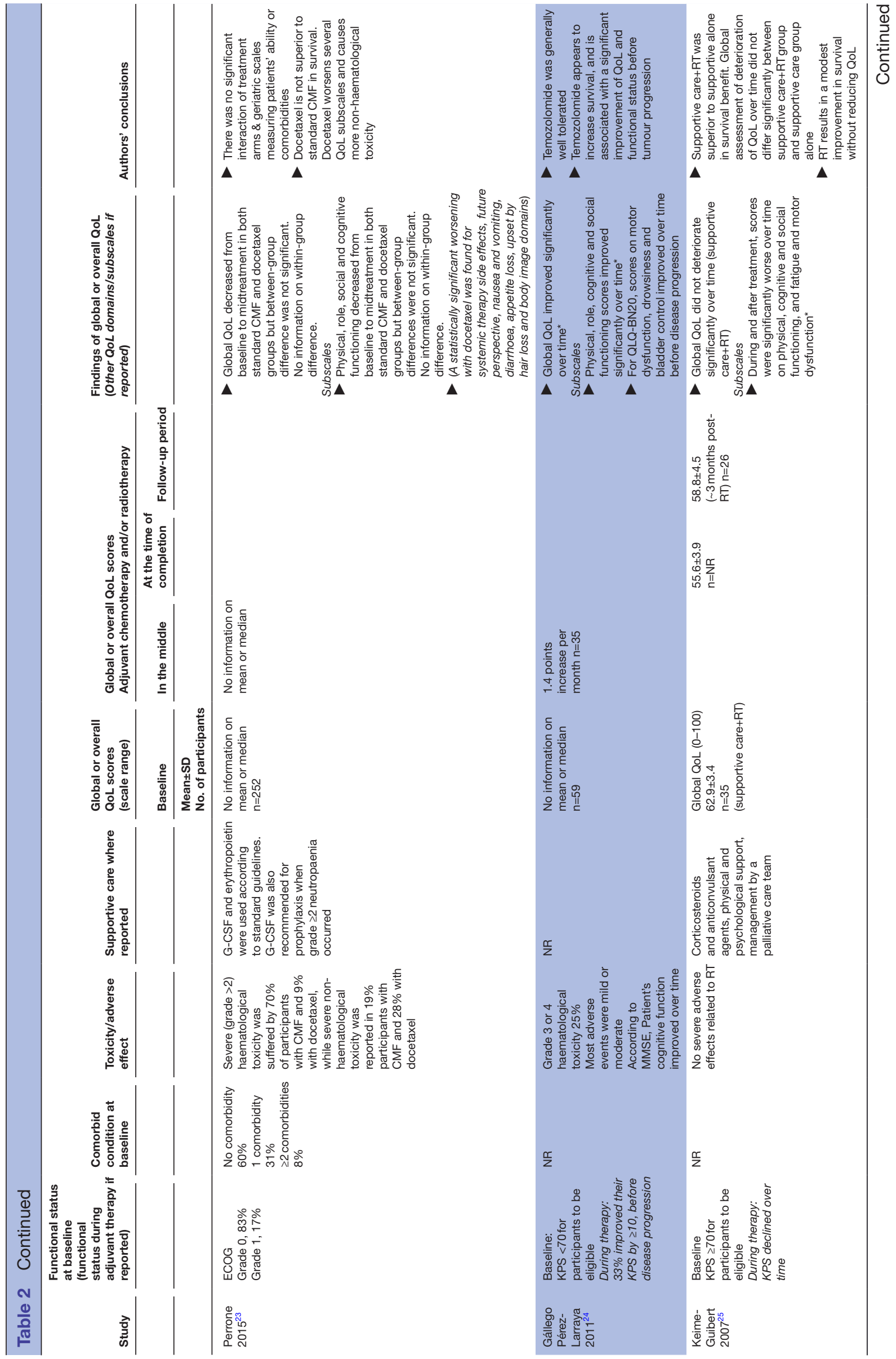




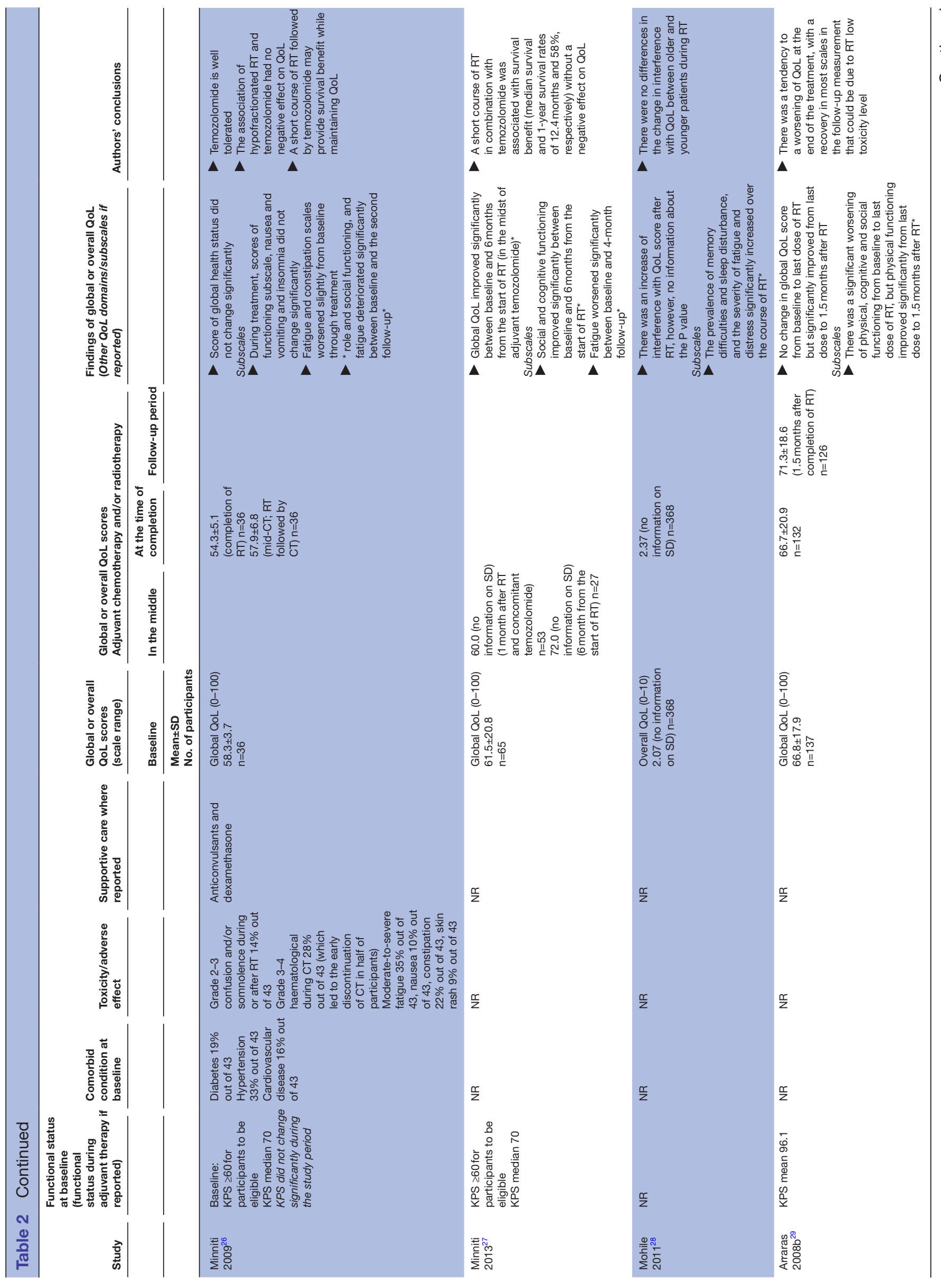




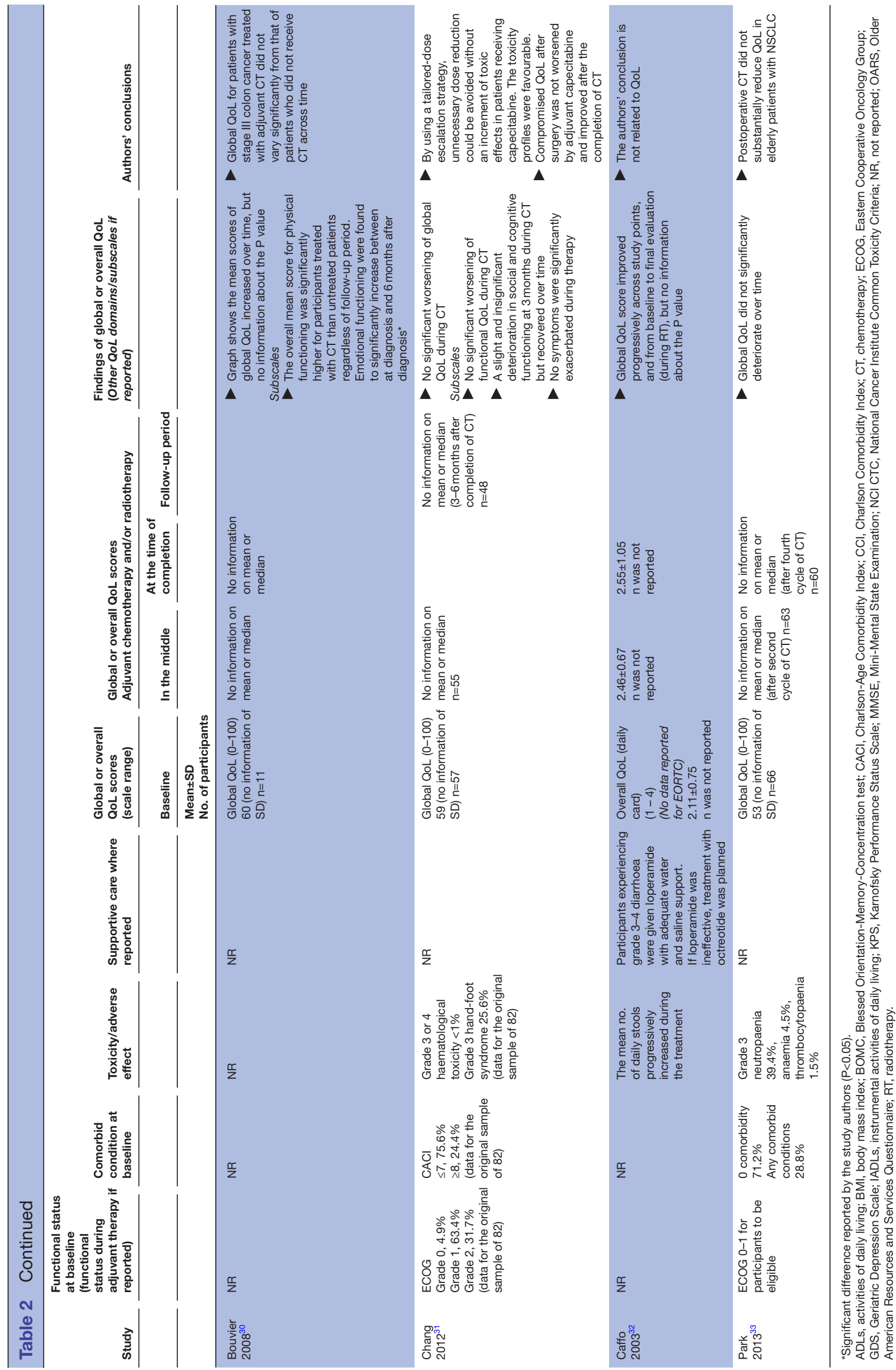




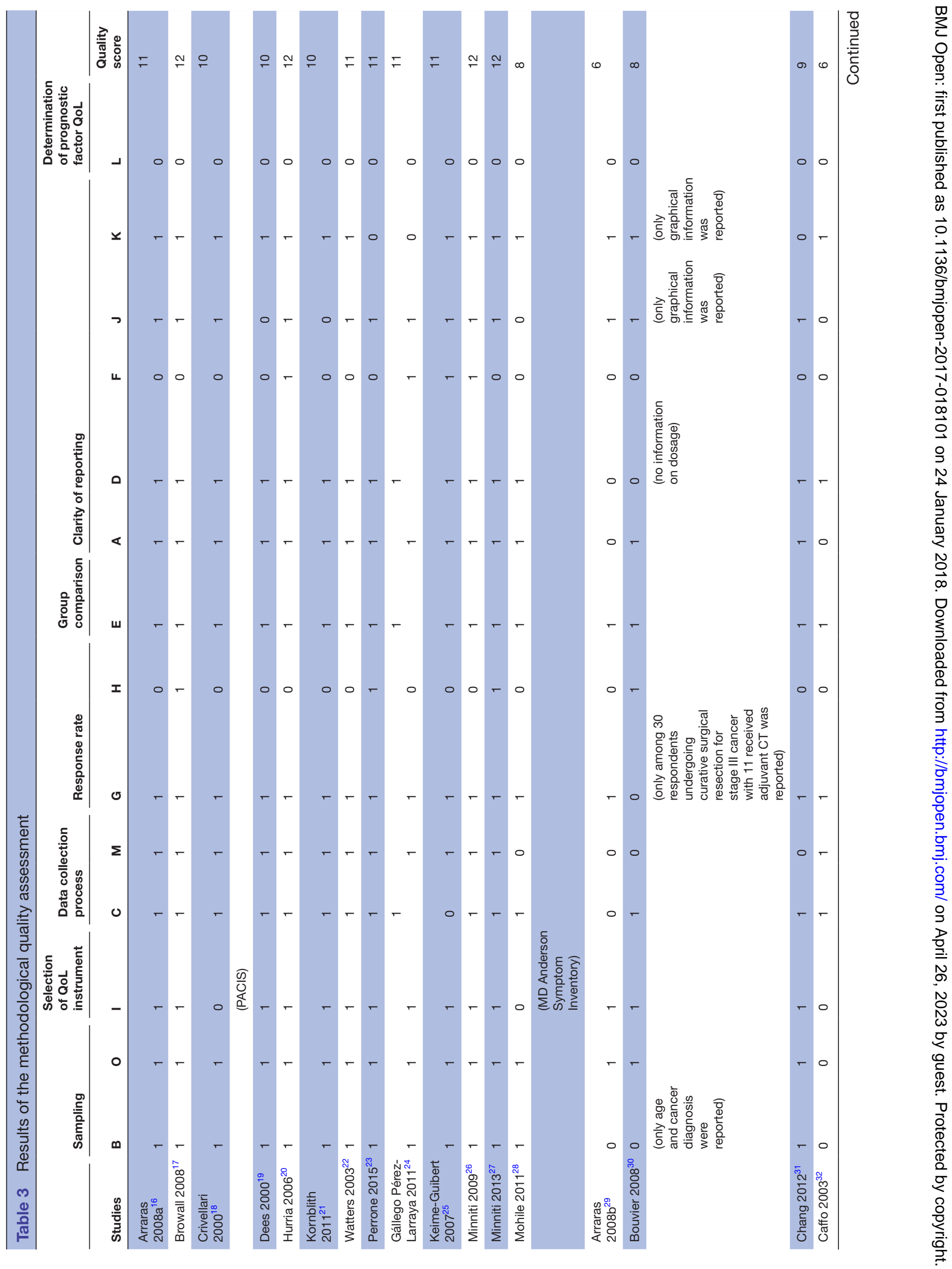



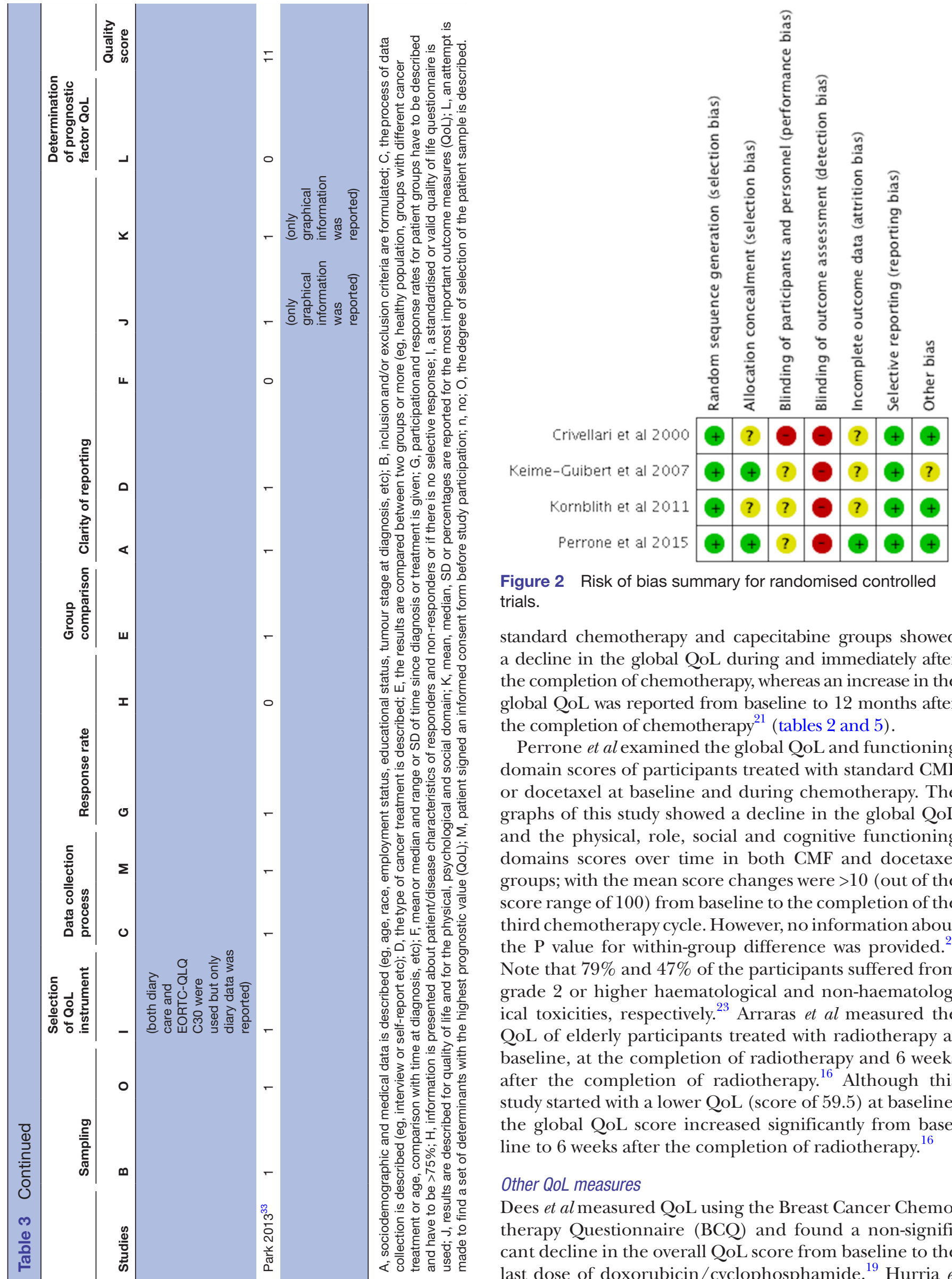

在

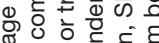

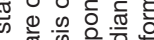

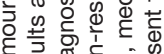

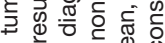

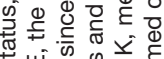

का एँ

Figure 2 Risk of bias summary for randomised controlled trials.

standard chemotherapy and capecitabine groups showed a decline in the global QoL during and immediately after the completion of chemotherapy, whereas an increase in the global QoL was reported from baseline to 12 months after the completion of chemotherapy ${ }^{21}$ (tables 2 and 5).

Perrone et al examined the global QoL and functioning domain scores of participants treated with standard CMF or docetaxel at baseline and during chemotherapy. The graphs of this study showed a decline in the global QoL and the physical, role, social and cognitive functioning domains scores over time in both CMF and docetaxel groups; with the mean score changes were $>10$ (out of the score range of 100) from baseline to the completion of the third chemotherapy cycle. However, no information about the $\mathrm{P}$ value for within-group difference was provided. ${ }^{23}$ Note that $79 \%$ and $47 \%$ of the participants suffered from grade 2 or higher haematological and non-haematological toxicities, respectively. ${ }^{23}$ Arraras et al measured the QoL of elderly participants treated with radiotherapy at baseline, at the completion of radiotherapy and 6 weeks after the completion of radiotherapy. ${ }^{16}$ Although this study started with a lower QoL (score of 59.5) at baseline, the global QoL score increased significantly from baseline to 6 weeks after the completion of radiotherapy. ${ }^{16}$

\section{Other QoL measures}

Dees et al measured QoL using the Breast Cancer Chemotherapy Questionnaire (BCQ) and found a non-significant decline in the overall QoL score from baseline to the last dose of doxorubicin/cyclophosphamide. ${ }^{19}$ Hurria $e t$ 
Table 4 Risk of bias summary for non-RCTs (ROBINS-I)

\begin{tabular}{|c|c|c|c|c|c|c|c|c|}
\hline & \multicolumn{2}{|c|}{ Preintervention } & \multirow{2}{*}{$\begin{array}{l}\text { At intervention } \\
\text { Bias in } \\
\text { classification } \\
\text { of } \\
\text { interventions }\end{array}$} & \multicolumn{4}{|c|}{ Postintervention } & \multirow[b]{2}{*}{ Overall risk of bias } \\
\hline Studies & $\begin{array}{l}\text { Bias due to } \\
\text { confounding }\end{array}$ & $\begin{array}{l}\text { Bias in } \\
\text { selection of } \\
\text { participants } \\
\text { into the } \\
\text { study }\end{array}$ & & $\begin{array}{l}\text { Bias due to } \\
\text { deviations } \\
\text { from intended } \\
\text { interventions }\end{array}$ & $\begin{array}{l}\text { Bias due to } \\
\text { missing data }\end{array}$ & $\begin{array}{l}\text { Bias in } \\
\text { measurement } \\
\text { of outcomes }\end{array}$ & $\begin{array}{l}\text { Bias in } \\
\text { selection of the } \\
\text { reported result }\end{array}$ & \\
\hline Arraras $2008 a^{16}$ & M & M & M & $M$ & $M$ & M & Unclear & $\begin{array}{l}\text { Low or moderate } \\
\text { risk if bias for all } \\
\text { domains }\end{array}$ \\
\hline Dees $2000^{19}$ & S & $\mathrm{S}$ & L & M & M & M & Unclear & $\begin{array}{l}\text { Serious risk of } \\
\text { bias in at least one } \\
\text { domain }\end{array}$ \\
\hline Hurria $2006^{20}$ & M & M & $\mathrm{L}$ & M & L & M & Unclear & $\begin{array}{l}\text { Low or moderate } \\
\text { risk if bias for all } \\
\text { domains }\end{array}$ \\
\hline $\begin{array}{l}\text { Gállego Pérez- } \\
\text { Larraya } 2011^{24}\end{array}$ & M & M & L & M & $\mathrm{S}$ & $S$ & Unclear & $\begin{array}{l}\text { Serious risk of } \\
\text { bias in at least one } \\
\text { domain }\end{array}$ \\
\hline Minniti $2009^{26}$ & M & M & L & M & L & M & Unclear & $\begin{array}{l}\text { Low or moderate } \\
\text { risk if bias for all } \\
\text { domains }\end{array}$ \\
\hline Minniti $2013^{27}$ & M & M & L & M & $\mathrm{S}$ & M & Unclear & $\begin{array}{l}\text { Serious risk of } \\
\text { bias in at least one } \\
\text { domain }\end{array}$ \\
\hline Mohile $2011^{28}$ & S & M & M & Unclear & L & M & Unclear & $\begin{array}{l}\text { Serious risk of } \\
\text { bias in at least one } \\
\text { domain }\end{array}$ \\
\hline Caffo $2003^{32}$ & S & S & L & Unclear & M & M & Unclear & $\begin{array}{l}\text { Serious risk of } \\
\text { bias in at least one } \\
\text { domain }\end{array}$ \\
\hline Park $2013^{33}$ & M & M & M & M & M & M & Unclear & $\begin{array}{l}\text { Low or moderate } \\
\text { risk if bias for all } \\
\text { domains }\end{array}$ \\
\hline
\end{tabular}

C, critical risk; L, low risk; M, moderate risk; S, serious risk.

$a l$ revealed no significant differences in overall or in physical, social and emotional well-being as measured by Functional Assessment of Cancer Therapy-Breast (FACT-B) from baseline to immediately after and 6 months after completion of an anthracycline-based, taxane-based or CMF regimen..$^{20}$ Note that $27 \%$ and $31 \%$ of the participants of this study suffered from grade 3 or 4 haematological and non-haematological toxicity, respectively. ${ }^{20}$ Crivellari et al reported increased global QoL scores as measured by the Perceived Adjustment to Chronic Illness Scale (PACIS), during and 18 months after the completion of the CMF regimen. ${ }^{18}$ Note that the participants of this study had a low QoL score of 59 at baseline. Fewer than $10 \%$ of the participants manifested grade 3 toxicity. ${ }^{18}$

\section{Glioblastoma}

All four studies were conducted on participants with glioblastoma treated with temozolomide ${ }^{24}$ or focal hypofractionated radiotherapy ${ }^{25}$ or combined radiotherapy and temozolomide. ${ }^{26} 27$ These studies assessed QoL using the EORTC QLQ-C30. Gállego Pérez-Larraya et al reported statistically significant improvements in the global score and the physical, role, cognitive and social domain scores during the course of temozolomide. ${ }^{24}$ Note that $25 \%$ of 


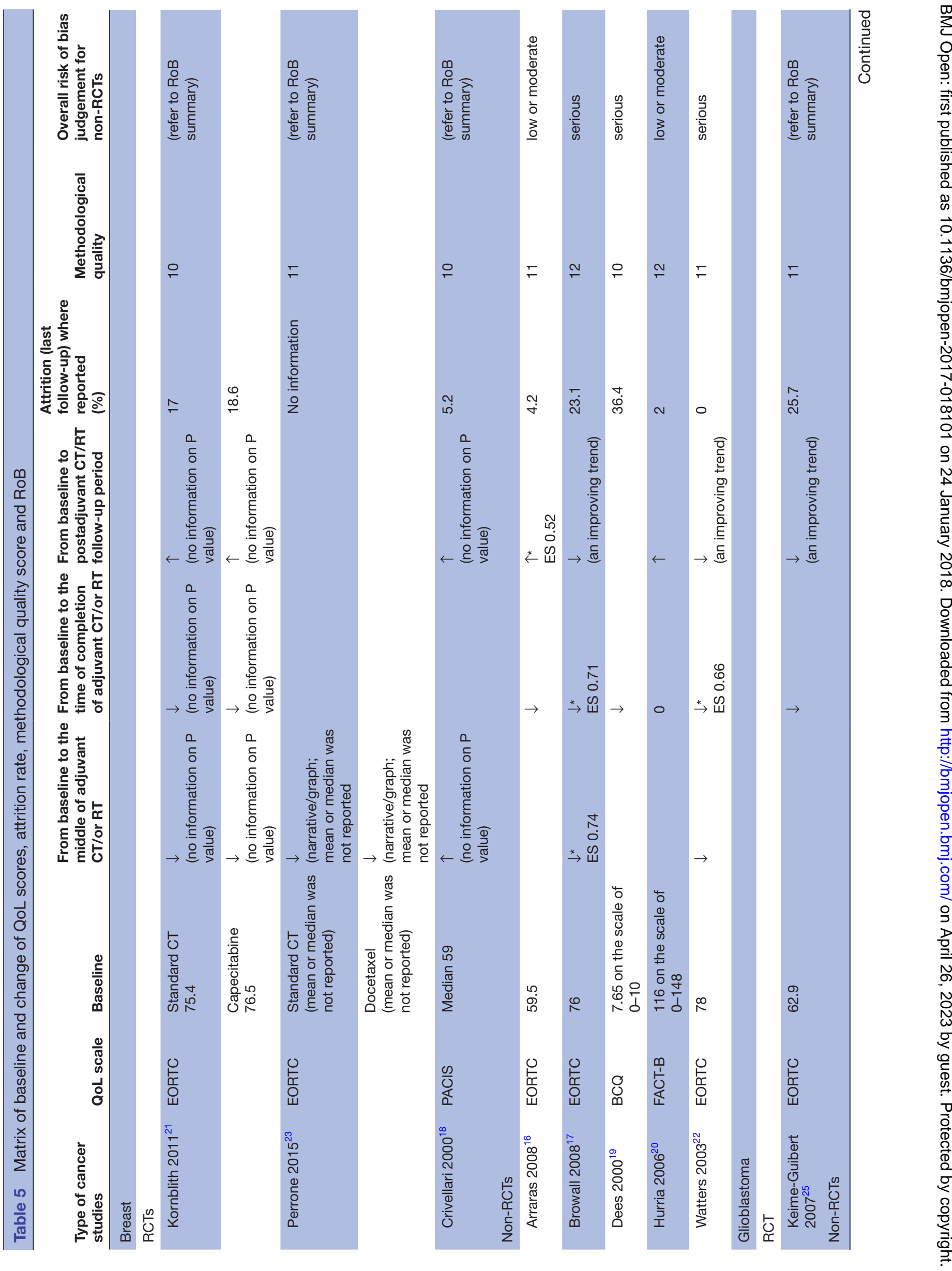



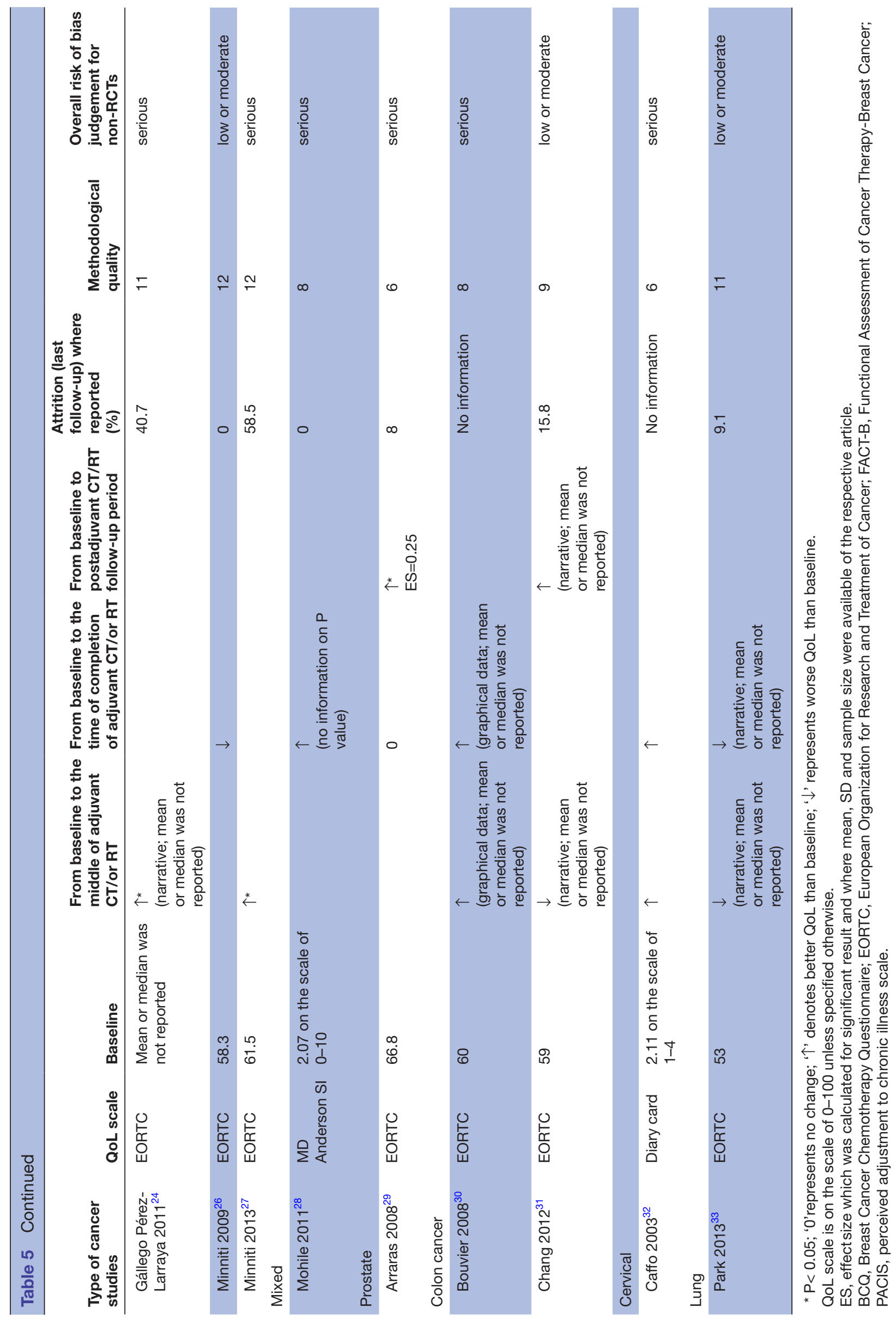
the participants manifested grade 3-4 haematological toxicity in this study. ${ }^{24}$ Minniti et al also showed statistically significant improvements in the global score and the social and cognitive domain scores from baseline to 6 months from the start of radiotherapy (which was during the course of temozolomide).$^{27}$ Both Keime-Gulbert et al. and Minniti et al reported a decline in the global QoL at the completion of focal hypofractionated radiotherapy. ${ }^{25}{ }^{26}$ With respect to the domain scores, these two studies reported statistically significantly lower scores for the physical, cognitive and social domains, and the physical, role and social domains, respectively, during and after radiotherapy than at baseline. ${ }^{25}{ }^{26}$ The participants in both studies were treated with corticosteroids and anticonvulsants as supportive care. Note that in the study by Minniti et al, the participants began with a lower QoL (score of 58.3) at baseline and that $14 \%$ of these participants developed grade 2 or 3 confusion and/or somnolence during or after radiotherapy. ${ }^{26}$

\section{Colon cancer}

Two studies measured the global QoL with the EORTC QLQ-C30 at baseline and during and after chemotherapy in participants with colon cancer. ${ }^{30}{ }^{31}$ In the study by Bouvier et al., the participants were treated with a fluorouracil/oxaliplatin/ capecitabine regimen. ${ }^{30}$ This study reported an increase in the global QoL scores over time; however, no information about the $\mathrm{P}$ value was provided. Chang et al. found no significant worsening of the global and functional QoL during capecitabine treatment. ${ }^{31}$

\section{Prostate cancer}

Arraras et almeasured QoL by using the EORTC QLQ-C30 in participants treated with radiotherapy for prostate cancer. ${ }^{29}$ No difference in the global QoL score was observed from baseline to the last dose of radiotherapy, whereas a statistically significantly higher QoL score was reported at 6 weeks after radiotherapy $(\mathrm{ES}, 0.25){ }^{29}$

\section{Lung cancer}

Park et al. measured the global QoL using the EORTC QLQ-C30 at baseline and 1 month after the completion of therapy with cisplatin plus vinorelbine or carboplatin plus paclitaxel in participants with resectable non-small cell lung carcinoma. ${ }^{33}$ In this study, the QoL score of 53 at baseline was low. No significant deterioration of the global QoL between baseline and the follow-up evaluation was observed. Severe haematological toxicity was manifested in $39 \%$ of the participants. ${ }^{33}$

\section{Other cancers}

Mohile et al studied different types of cancer, and QoL was measured before and after radiotherapy using an item of interference with overall QoL together with the modified MD Anderson Symptom Inventory. ${ }^{25}$ In this study, the overall QoL score of 2.07 on the scale of 10 at baseline was low. A slightly higher overall QoL score was shown at the completion of radiotherapy (score of 2.37); however, no information about the $\mathrm{P}$ value was reported. ${ }^{28}$

\section{DISCUSSION}

In the context of cancer, QoL by its nature is a patient's overall appraisal of the effect of cancer and its treatment. It is a patient-centred, relevant and key clinical parameter that can assist and support clinicians in setting goals and mapping avenues for effective and tolerable cancer treatment regimens beyond extending patient survival. Although the 18 studies included in this systematic review had somewhat heterogeneous study designs, cancer populations and measurement scales and reporting parameters of QoL to permit data pooling for a meta-analysis and precise estimation, our results provide some insights that will contribute to a better understanding of the effects of adjuvant chemotherapy and/or radiotherapy on the QoL of elderly patients aged 65 years or above. Our review suggests that QoL during and after adjuvant chemotherapy and/or radiotherapy is maintained or improved in most patients with solid tumours.

For elderly patients with breast cancer, the non-significant negative change in the global or overall QoL was transient (during and immediately after chemotherapy or radiotherapy), as measured by the EORTC QLO-C30, FACT-B and BCQ. No lasting adverse effect on QoL was observed after completion of the adjuvant treatment (overall low or moderate to serious RoB). ${ }^{16-2123}$ Browall et al and Watters et al revealed an initial statistically significant decline (moderate ES), followed by progressive improvement in global QoL scores from baseline to 4-6 months after chemotherapy (overall serious RoB). The role and social domains of QoL was mostly impaired immediately after the completion of chemotherapy. ${ }^{17} 22$

Another finding of this review is the significant increase in the global QoL during the course of temozolomide treatment in elderly patients with glioblastoma (overall low or moderate to serious RoB), ${ }^{24}{ }^{27}$ but a decreasing trend in QoL immediately after the completion of radiotherapy and 3 months after radiotherapy. ${ }^{25}{ }^{26}$ Note that the studies by Gállego Pérez-Larraya et al and Minniti et al had substantial amounts of missing data $(>40 \%)$, mainly because of the rapid progression of the disease in the glioblastoma population. However, the approach of complete case evaluation used in the final QoL analysis could have led to a systematic bias in the estimation of the true effect of adjuvant therapy on QoL towards high QoL scores. Therefore, some caution should be taken in the interpretation of the significant QoL improvement during the course of adjuvant therapy of elderly patients with glioblastoma. Nevertheless, attrition bias is always an issue in clinical trials involving QoL assessments and longitudinal follow-ups.

Adjuvant chemotherapy or radiotherapy also does not seem to compromise the QoL of elderly patients with prostate, colon or cervical cancer. This review shows a uniform trend of stable or improved global or overall QoL over the course of adjuvant therapy and at follow-up evaluations across the studies with prostate, colon or cervical cancer population (overall serious $\mathrm{RoB}){ }^{28-30} 32 \mathrm{~A}$ decreasing trend in global or overall 
QoL during and immediately after the completion of cisplatin or carboplatin treatment in elderly patients with lung cancer was reported in one study (overall low-to-moderate RoB). ${ }^{33}$

We expected altered functional status, comorbidities, adverse effects, haematological status and liver and renal functional status to covary with the effect of adjuvant therapy on QoL and hence, to be plausible confounding factors in the geriatric and adjuvant settings. However, as is the case in non-RCT settings, adjuvant therapy was allocated during the course of usual treatment decisions. The non-RCTs included in this review might suffer from the methodological drawbacks of uncontrolled confounding factors at baseline and even during the follow-up. Because no attempt was made to control confounding factors with a stratified design and analysis, caution is warranted in the interpretation of the results. Nevertheless, we found it difficult to discern whether the short period of QoL impairment and the stable or improved QoL over the course of adjuvant therapy and after treatment were due to the relatively low treatment toxicities, the relatively few morbid conditions or other reasons. The fact that, where reported, the QoL of elderly patients was maintained or improved over the course of treatment, despite the haematological toxicity across studies, ${ }^{20} 232433$ suggests that stable or improved QoL is unlikely to be attributable to relatively low treatment toxicity. Alternatively, elderly patients with cancer who undergo adjuvant therapy may experience adverse effects but can tolerate them with a limited effect on their QoL. This finding may also be attributed to the tendency of certain elderly patients to complain less and endure the relatively high morbidity associated with adverse effects. ${ }^{5}$ Elderly patients may also have a positive perception of the adjuvant therapy and may adjust better to the treatment. Stone et al examined the association between global well-being and the age profile of 340847 people and showed that people aged over 50 years have increased global well-being and positive emotions even in the face of a decline in the physical health. ${ }^{34}$ Another possible explanation for the stable or improved QoL could be the response shift phenomenon, in which patients experience a shift in how they appreciate their QoL over time as a result of the changes in their internal standards of measurement, values or definition of QoL. ${ }^{35}{ }^{36}$ A future qualitative study is needed to explore in detail QoL perception and experiences in adjuvant settings and adjustment to the treatment among elderly patients with cancer. Nevertheless, for studies that reported a stable global or overall QoL (ie, no difference in the means) across time, a small sample size and attrition bias might limit the statistical power to detect the differences between the baseline and the follow-up evaluations. ${ }^{19} 21232531$ It could also be argued that another possible bias was the poor sensitivity of the generic QoL measures to tap dimensions of health status that are particularly salient to elderly patients with cancer during adjuvant therapy. While we cannot rule out the possible bias, in future clinical trials and observational studies attempts should be made to use geriatric oncology-specific QoL measures such as EORTC-QLQ-ELD14 to validate the review results. ${ }^{37}$ Furthermore, the samples of the included studies appear highly functional at baseline,$^{16-2325-33}$ so these studies may be subject to a selection bias pertaining to under-representation of less healthy older patients and those with limited expectations of treatment benefits. ${ }^{3}$

\section{CONCLUSIONS}

This review suggests that a negative change in QoL was short-lived during adjuvant chemotherapy for some elderly patients with breast cancer. Adjuvant chemotherapy and radiotherapy may not have detrimental effects on global or overall QoL and other QoL domains in most elderly patients with solid tumours. These findings could be translated to help future elderly patients better understand the impact of adjuvant therapy on their QoL, and hence make better treatment decisions. Nevertheless, our review results should be viewed with caution because of RoB within and across the included studies. In addition, heterogeneity in study design and measurement of QoL, and lack of availability of data limit the pooling of data for meta-analysis and affect the robustness of the evidence synthesis. An attempt was made to contact the study authors for data, but without success. There is also a possibility of incompleteness of evidence because of unclear bias of the selection of reported result and the search of this review did not include grey literature, unpublished studies, ongoing clinical trials and theses and dissertations. Larger and well-designed studies of elderly patients in different cancer settings are warranted to validate these review results and to further build evidence to advance the current knowledge base. These studies should include and stratify elderly patients by functional status, comorbid conditions, geriatric syndromes and prognosis to be more representative of the real-world population and improve the research validity. Future studies should also include a detailed profile of the cytotoxic effects of chemotherapy and radiotherapy to allow a full exploration of the direct and indirect effects of adjuvant therapy on QoL. In future systematic reviews, if sufficient data are available, meta-regression should also be conducted to examine the association and interaction between the confounding factors and the QoL.

Acknowledgements The authors would like to thank Teo Desrene Rui Ling (TDRL), Research Assistant, for her work in literature search, eligibility assessments and study selection.

Contributors CKK-F, KR contributed to the conception or design of the work, and analysis and interpretation of data. CKK- $\mathrm{F}$ is responsible for drafting the manuscript. KR critically reviewed and revised the manuscript for important intellectual content. LEY-T contributed to the acquisition of data and critical revision of the manuscript for intellectual content. CKK-F, LEY-T, KR provided final approval of the version to be published.

Funding This research received no specific grant from any funding agency in the public, commercial or not-for-profit sectors. 
Competing interests None declared.

Patient consent Not required.

Provenance and peer review Not commissioned; externally peer reviewed.

Data sharing statement № additional data are available.

Open Access This is an Open Access article distributed in accordance with the Creative Commons Attribution Non Commercial (CC BY-NC 4.0) license, which permits others to distribute, remix, adapt, build upon this work non-commercially, and license their derivative works on different terms, provided the original work is properly cited and the use is non-commercial. See: http://creativecommons.org/ licenses/by-nc/4.0/

(C) Article author(s) (or their employer(s) unless otherwise stated in the text of the article) 2018. All rights reserved. No commercial use is permitted unless otherwise expressly granted.

\section{REFERENCES}

1. Yancik R, Ries LA. Cancer in older persons: an international issue in an aging world. Semin Oncol 2004;31:128-36.

2. Finkel T, Serrano M, Blasco MA. The common biology of cancer and ageing. Nature 2007;448:767-74.

3. Hurria A, Levit LA, Dale W, et al. Improving the evidence base for treating older adults with cancer: american society of clinical oncology statement. J Clin Oncol 2015;33:3826-33.

4. International society of geriatric oncology. 2015 http://www.siog.org/.

5. Reimer T, Gerber B. Quality-of-life considerations in the treatment of early-stage breast cancer in the elderly. Drugs Aging 2010;27:791-800

6. Wedding U, Pientka L, Höffken K. Quality-of-life in elderly patients with cancer: a short review. Eur J Cancer 2007;43:2203-10.

7. Extermann M, Reich RR, Sehovic M. Chemotoxicity recurrence in older patients: Risk factors and effectiveness of preventive strategies-a prospective study. Cancer 2015;121:2984-92.

8. Joerger M, Thürlimann B, Savidan A, et al. Treatment of breast cancer in the elderly: a prospective, population-based Swiss study. J Geriatr Oncol 2013;4:39-47.

9. Taphoorn MJ, Henriksson R, Bottomley A, et al. Health-related quality of life in a randomized phase iii study of bevacizumab, temozolomide, and radiotherapy in newly diagnosed glioblastoma. $J$ Clin Oncol 2015;33:2166-75.

10. de Haes JC, Curran D, Aaronson NK, et al. Quality of life in breast cancer patients aged over 70 years, participating in the EORTC 10850 randomised clinical trial. Eur J Cancer 2003;39:945-51.

11 Mols F, Vingerhoets AJ, Coebergh JW, et al. Quality of life among long-term breast cancer survivors: a systematic review. Eur J Cancer 2005;41:2613-9.

12 Cornish D, Holterhues C, van de Poll-Franse LV, et al. A systematic review of health-related quality of life in cutaneous melanoma. Ann Oncol 2009;20(Suppl 6):vi51-8.

13 Borghouts JA, Koes BW, Bouter LM. The clinical course and prognostic factors of non-specific neck pain: a systematic review. Pain 1998;77:1-13.

14 Sterne JA, Hernán MA, Reeves BC, et al. ROBINS-I: a tool for assessing risk of bias in non-randomised studies of interventions. BMJ 2016;355:i4919.

15. Higgins IPT, Altman DG, Gotzsche PC, et al. Cochrane Bias Methods Group Cochrane Statistical Methods Group. The Cochrane Collaboration's tool for assessing risk of bias in randomised trials. BMJ 2011;343:d5928.

16. Arraras JI, Manterola A, Domínguez MA, et al. Impact of radiotherapy on the quality of life of elderly patients with localized breast cancer. A prospective study. Clin Transl Oncol 2008;10:498-504.

17. Browall MM, Ahlberg KM, Persson LO, et al. The impact of age on Health-Related Quality of Life (HRQoL) and symptoms among postmenopausal women with breast cancer receiving adjuvant chemotherapy. Acta Oncol 2008;47:207-15.

18. Crivellari D, Bonetti M, Castiglione-Gertsch M, et al. Burdens and benefits of adjuvant cyclophosphamide, methotrexate, and fluorouracil and tamoxifen for elderly patients with breast cancer: the International Breast Cancer Study Group Trial VII. J Clin Oncol 2000;18:1412-22.

19. Dees EC, O'Reilly S, Goodman SN, et al. A prospective pharmacologic evaluation of age-related toxicity of adjuvant chemotherapy in women with breast cancer. Cancer Invest 2000;18:521-9.

20. Hurria A, Hurria A, Zuckerman E, et al. A prospective, longitudinal study of the functional status and quality of life of older patients with breast cancer receiving adjuvant chemotherapy. J Am Geriatr Soc 2006:54:1119-24.

21. Kornblith AB, Lan L, Archer L, et al. Quality of life of older patients with early-stage breast cancer receiving adjuvant chemotherapy: a companion study to cancer and leukemia group B 49907. J Clin Oncol 2011;29:1022-8.

22. Watters JM, Yau JC, O'Rourke K, et al. Functional status is well maintained in older women during adjuvant chemotherapy for breast cancer. Ann Oncol 2003;14:1744-50.

23. Perrone F, Nuzzo F, Di Rella F, et al. Weekly docetaxel versus CMF as adjuvant chemotherapy for older women with early breast cancer: final results of the randomized phase III ELDA trial. Ann Oncol 2015;26:675-82.

24. Gállego Pérez-Larraya J, Ducray F, Chinot O, et al. Temozolomide in elderly patients with newly diagnosed glioblastoma and poor performance status: an ANOCEF phase II trial. J Clin Oncol 2011;29:3050-5.

25. Keime-Guibert F, Chinot O, Taillandier L, et al. Radiotherapy for glioblastoma in the elderly. N Engl J Med 2007;356:1527-35.

26. Minniti G, De Sanctis V, Muni R, et al. Hypofractionated radiotherapy followed by adjuvant chemotherapy with temozolomide in elderly patients with glioblastoma. J Neurooncol 2009;91:95-100.

27. Minniti G, Scaringi C, Baldoni A, et al. Health-related quality of life in elderly patients with newly diagnosed glioblastoma treated with short-course radiation therapy plus concomitant and adjuvant temozolomide. Int J Radiat Oncol Biol Phys 2013;86:285-91.

28. Mohile SG, Heckler C, Fan L, et al. Age-related differences in symptoms and their interference with quality of life in 903 cancer patients undergoing radiation therapy. $J$ Geriatr Oncol 2011;2:225-32.

29. Arraras Urdaniz JI, Villafranca Iturre E, Arias de la Vega F, et al. The eortc quality of life questionnaire QLQ-C30 (version 3.0). Validation study for Spanish prostate cancer patients. Arch Esp Urol 2008;61:949-54.

30. Bouvier AM, Jooste V, Bonnetain F, et al. Adjuvant treatments do not alter the quality of life in elderly patients with colorectal cancer: a population-based study. Cancer 2008;113:879-86

31. Chang HJ, Lee KW, Kim JH, et al. Adjuvant capecitabine chemotherapy using a tailored-dose strategy in elderly patients with colon cancer. Ann Oncol 2012;23:911-8.

32. Caffo O, Amichetti M, Mussari S, et al. Physical side effects and quality of life during postoperative radiotherapy for uterine cancer. Prospective evaluation by a diary card. Gynecol Oncol 2003;88:270-6

33. Park S, Kim IR, Baek KK, et al. Prospective analysis of quality of life in elderly patients treated with adjuvant chemotherapy for non-smallcell lung cancer. Ann Oncol 2013;24:1630-9.

34. Stone AA, Schwartz JE, Broderick JE, et al. A snapshot of the age distribution of psychological well-being in the United States. Proc Natl Acad Sci U S A 2010;107:9985-90.

35. Cemal Y, Jewell S, Albornoz CR, et al. Systematic review of quality of life and patient reported outcomes in patients with oncologic related lower extremity lymphedema. Lymphat Res Biol 2013;11:14-19.

36. Ring L, Höfer S, Heuston F, et al. Response shift masks the treatment impact on patient reported outcomes (PROs): the example of individual quality of life in edentulous patients. Health Qual Life Outcomes 2005;3:55.

37. Wheelwright S, Darlington AS, Fitzsimmons D, et al. International validation of the EORTC QLQ-ELD14 questionnaire for assessment of health-related quality of life elderly patients with cancer. $\mathrm{Br} J$ Cancer 2013;109:852-8.

38. Aaronson NK, Ahmedzai S, Bergman B, et al. The European Organization for Research and Treatment of Cancer QLQ-C30: a quality-of-life instrument for use in international clinical trials in oncology. J Natl Cancer Inst 1993;85:365-76.

39. Sprangers MA, Groenvold M, Arraras JI, et al. The European Organization for Research and Treatment of Cancer breast cancerspecific quality-of-life questionnaire module: first results from a three-country field study. J Clin Oncol 1996;14:2756-68.

40. Hürny $\mathrm{C}$, Bernhard J, Bacchi M, et al. The Perceived Adjustment to Chronic IIIness Scale (PACIS): a global indicator of coping for operable breast cancer patients in clinical trials. Swiss Group for Clinical Cancer Research (SAKK) and the International Breast Cancer Study Group (IBCSG). Support Care Care 1993;1:200-8.

41. Levine MN, Guyatt GH, Gent M, et al. Quality of life in stage Il breast cancer: an instrument for clinical trials. J Clin Oncol 1988:6:1798-810.

42. Brady MJ, Cella DF, Mo F, et al. Reliability and validity of the functional assessment of cancer therapy-breast quality-of-life instrument. J Clin Oncol 1997:15:974-86. 
43. Ware JE, Sherbourne CD. The MOS 36-item short-form health survey (SF-36). I. Conceptual framework and item selection. Med Care 1992;30:473-83.

44. Taphoorn MJ, Claassens L, Aaronson NK, et al. An international validation study of the EORTC brain cancer module (EORTC QLQBN20) for assessing health-related quality of life and symptoms in brain cancer patients. Eur J Cancer 2010;46:1033-40.

45. Cleeland CS, Mendoza TR, Wang XS, et al. Assessing symptom distress in cancer patients: the M.D. Anderson Symptom Inventory. Cancer 2000;89:1634-46.
46. Fraser SC, Ramirez AJ, Ebbs SR, et al. A daily diary for quality of life measurement in advanced breast cancer trials. $\mathrm{Br} \mathrm{J}$ Cancer 1993;67:341-6.

47. Bergman B, Aaronson NK, Ahmedzai S, et al. The EORTC QLQLC13: a modular supplement to the EORTC Core Quality of Life Questionnaire (QLQ-C30) for use in lung cancer clinical trials. EORTC Study Group on Quality of Life. Eur J Cancer 1994;30A:635-42. 